\title{
La fase preanalítica en la acreditación de los laboratorios clínicos: una mirada al mejoramiento de la competencia técnica
}

\author{
Preanalytical phase in the accreditation of clinical laboratories: \\ a look to the improvement of technical competence
}

Más allá de la habilitación y la certificación de los laboratorios clínicos como prestadores de servicios de salud con un sistema de gestión de calidad establecido, la búsqueda de las acreditaciones internacionales que avalen, respalden y soporten la competencia técnica de los laboratorios clínicos se ha convertido en una herramienta que, adicional a la obtención de un certificado, ha permitido el mejoramiento continuo. Esto, gracias a que los sistemas de acreditación establecen pautas específicas para la estandarización y el control de todas las fases de la prestación de los servicios de los laboratorios, en donde se incluyen aquellas más susceptibles al error humano, como la fase preanalítica.

Los servicios de habilitación de los laboratorios clínicos, al avalarlos como prestadores de servicios de salud en el territorio colombiano, establecen los requisitos mínimos que deben cumplir para su funcionamiento con énfasis en la capacidad técnico-administrativa, la suficiencia patrimonial y financiera, la capacidad tecnológica y científica, el recurso humano, las instalaciones físicas, la dotación y el mantenimiento, la gestión de medicamentos y dispositivos, los procesos prioritarios asistenciales, la gestión de riesgos y la seguridad del paciente, entre otros [1]. A su vez, los servicios de certificación, como la ISO 9001, a pesar de no ser específicos de los laboratorios clínicos, permiten la estructuración de un sistema de gestión de la calidad efectivo, cuya decisión estratégica de implementación ayuda a mejorar el desempeño y el desarrollo sostenible de las instituciones [2].

Luego de establecer un sistema de gestión de la calidad fortalecido se requiere mejorar y avalar la competencia técnica de los laboratorios clínicos a partir de una evaluación más rigurosa de su quehacer, por lo que la acreditación se convierte en una oportunidad invaluable para el mejoramiento de los mismos. Entre los entes de acreditación, específicos de los laboratorios clínicos, se destacan la Organización Internacional de Normalización (ISO), con su norma NTCISO 15189 [3], y el Colegio Americano de Patólogos (CAP), con su programa de acreditación internacional [4].

La NTC-ISO 15189, en su numeral 5.4. Procedimientos preanalíticos, hace referencia específica al manejo de la información de los pacientes y los usuarios, la información de la solicitud requerida y los procesos de toma y manipulación de la muestra primaria, transporte, preparación y almacenamiento previos al análisis [3], lo que brinda las pautas básicas para que los laboratorios clínicos estandaricen y controlen todos los aspectos relacionados con la toma de muestra. Incluso, en esta norma se describen los lineamientos de la orden médica y el uso y la recolección de los datos de los pacientes y usuarios, por lo que el alcance de su aplicación, de forma rigurosa y metódica por parte de los laboratorios, contribuye a disminuir las tasas de error en la fase preanalítica. 
Por su parte, el CAP, en sus instrucciones para la toma y la manipulación de las muestras, en las que hace énfasis en contar con un manual de toma de muestras, la marcación de los contenedores primarios y secundarios de la muestra, los criterios de rechazo de la muestra [4], entre otros aspectos específicos de cada sección del laboratorio, da las directrices de lo que el laboratorio debe proveer para garantizar una toma de muestra adecuada, independiente de quién la realice. Igualmente, hace hincapié en la identificación positiva de las muestras y la trazabilidad de las mismas durante toda la fase preanalítica.

Es así como los entes acreditadores, además de establecer pautas sobre la alta gerencia, el sistema de gestión de la calidad y los procesos misionales de los laboratorios, se enfocan en todos los aspectos que impactan en la calidad de los resultados emitidos por los laboratorios clínicos [5], entre los cuales se destaca la fase preanalítica. A su vez, cada ente acreditador, al tener enfoques diferentes, hará mayor o menor énfasis sobre ciertos procedimientos inherentes a la toma de la muestra, que mejorarán las competencias técnicas de los laboratorios clínicos, siempre y cuando estos asuman los procesos de acreditación como una oportunidad de mejora.

En conclusión, los laboratorios clínicos, al estar inmersos en la práctica médica y proveer la información necesaria para la toma de decisiones médicas que generan un impacto directo en el diagnóstico, el tratamiento y el seguimiento de los pacientes, deben garantizar que los resultados emitidos cumplan con altos estándares de calidad y que ello se logre desde que el paciente acude al laboratorio clínico con una orden médica para la toma de muestras. Con base en lo anterior, la participación de todo el personal de laboratorio en el inicio de los procesos de acreditación, el cual es un proceso netamente voluntario en nuestro país y que en gran medida actúa como un «visto bueno» para las prácticas y las competencias técnicas de los laboratorios, se convertirá en el camino hacia la excelencia, el aseguramiento de la trazabilidad de los resultados y la estrategia para que los laboratorios clínicos ofrezcan un servicio de alta calidad, confiabilidad y utilidad médica.

\section{Natolio Guevara A.}

\section{Natalia María Guevara Arismendy}

MB, MSc en Microbiología y Bioanálisis, énfasis Hematología

Bacterióloga, Laboratorio Clínico Hematológico

Medellín, Colombia, octubre 2016

\section{Bibliografía}

1. República de Colombia, Ministerio de Salud y Protección Social. Resolución Número 00002003 de 2014. Disponible: https://www.minsalud.gov. co/Normatividad_Nuevo/Resoluci\%C3\%B3n\%20 2003\%20de\%202014.pdf. Consultado: oct 2016.

2. Instituto Colombiano de Normas Técnicas y Certificación. Norma Técnica Colombiana NTCISO 9001: Sistemas de gestión de la calidad. Requisitos (ed Cuarta actualización editada): ICONTEC; 2015.

3. Instituto Colombiano de Normas Técnicas y Certificación. NTC-ISO 15189. Laboratorios clíni- cos. Requisitos generales para la calidad y la competencia: ICONTEC; 2014.

4. College of American Pathologists, CAP Accreditation Program. Master All Common Checklist. 2015. Disponible: http://www.cap.org/ ShowProperty? nodePath =/UCMCon/Contribution\%20Folders/DctmContent/education/OnlineCourseContent/2016/LAP-TLTM/resources/AC-allcommon.pdf. Consultado: oct 2016.

5. Izquierdo-Álvarez S. Acreditación: el camino hacia la excelencia en ellaboratorio clínico. Rev Calidad Asistencial 2015; 30: e1-e3. 